\title{
Role of asymmetric dimethylarginine in rat acute lung injury induced by acute ischemic kidney injury
}

\author{
TAO MA, XIAOWEI LIU and ZHI LIU \\ Department of Emergency Medicine, The First Affiliated Hospital of China Medical University, \\ Shenyang, Liaoning 110001, P.R. China
}

Received December 2, 2013; Accepted July 21, 2014

DOI: $10.3892 / \mathrm{mmr} .2015 .3619$

\begin{abstract}
The aim of the present study was to establish a rat model of acute ischemic kidney injury by continually occluding the bilateral renal artery and renal veins, as well as to examine the role of asymmetric dimethylarginine (ADMA) in acute lung injury (ALI) induced by acute kidney injury (AKI). In total, 45 male Wistar rats were randomly divided into the control, AKI and AKI + SB203580 groups. The enzyme-linked immunosorbent assay method was used to assess the plasma levels of ADMA in each group. The activation of p38 mitogen-activated protein kinase (MAPK) and heat shock protein 27 (HSP27) was detected using western blot analysis. Immunofluorescence analyses of filamentous actin (F-actin) and globular actin (G-actin) were performed. The levels of plasma ADMA and the permeability index in the AKI group were significantly higher than those in the control and AKI + SB203580 groups. The expression of phosphorylated p38 (p-p38) and p-HSP27 was significantly higher in the AKI and AKI + SB203580 groups compared with the control group. The AKI group also demonstrated a higher expression of p-p38 and p-HSP27 than the AKI + SB203580 group. The expression of F-actin was more marked in the AKI group than in the control and AKI + SB203580 groups. The $\mathrm{F}-/ \mathrm{G}$-actin ratio in the AKI group was also higher compared with that in the control and AKI + SB203580 groups. The results from the present study indicated that plasma ADMA levels may be a good biomarker for AKI-induced ALI. In addition, the p38 MAPK/HSP27 pathway is important in modulating the levels of ADMA.
\end{abstract}

Correspondence to: Professor Zhi Liu, Department of Emergency Medicine, The First Affiliated Hospital of China Medical University, 155 Nanjing Street, Heping, Shenyang, Liaoning 110001, P.R. China E-mail: cmu1h_lz@163.com

Key words: acute kidney injury, acute lung injury, asymmetric dimethylarginine, p38 mitogen-activated protein kinase, heat shock protein 27

\section{Introduction}

Acute kidney injury (AKI) is a relatively common complication in hospitalized patients with an overall mortality rate of $\sim 30 \%(1,2)$. AKI is associated with a high mortality rate and may lead to increased hospital costs (3). Previous studies have demonstrated that $5-10 \%$ of patients admitted to the intensive care unit (ICU) developed AKI and required renal replacement therapy (RRT) $(4,5)$. Although the mortality rate of AKI has been reduced since the application of RRT, its mortality rate remains as high as $40-70 \%$ (6). Among all the internal organs, the lung is most easily damaged by ischemic injuries due to the extensive vascular network in the lung mesenchyme (7). It is well established that cardiac and non-cardiac acute lung injuries (ALI) are the main causes of mortality in patients with AKI (8). Although the exact pathophysiology of AKI-induced ALI remains to be elucidated, the general mechanisms underlying AKI-induced ALI are well understood, including inflammation, activation of soluble and cellular factors as well as neurohumoral and hemodynamic alterations (9).

Asymmetric dimethylarginine (ADMA) is an endogenous nitric oxide (NO) synthase inhibitor, which may affect the biological activity of NO (10). ADMA is a naturally occurring amino acid that is found in tissues and cells, circulates in the plasma and is expelled in the urine (11). ADMA is eliminated through metabolic degradation and renal excretion (12). Increased blood concentrations of ADMA may contribute to endothelial dysfunction, thereby linking to the development and progression of chronic and acute kidney diseases (13). Activation of the p38 mitogen-activated protein kinase (MAPK)/heat shock protein 27 (HSP27) pathway by elevated ADMA has been verified, and is involved in endothelial dysfunction and abnormal permeability $(10,14)$. Thus, the excessive accumulation of ADMA accompanied by elevated HSP27 levels provides a potential explanation for the occurrence of AKI-induced ALI. The aim of the present study was to establish a rat model of acute ischemic kidney injury by continually occluding the bilateral renal artery and renal veins. In addition, the roles of ADMA in the development of AKI-induced ALI were examined.

\section{Materials and methods}

Study approval. The animal use protocol was reviewed and approved by the Institutional Animal Care and Use Committee 
of the First Affiliated Hospital of China Medical University (Shenyang, Liaoning, China). The present study was performed strictly in accordance with the recommendations of the Guide for the Care and Use of Laboratory Animals of the National Institutes of Health (Bethesda, MA, USA).

Animal models. In total, 45 male Wistar rats, weighing 300-320 g, were provided by the Experimental Animal Center of the China Medical University. A solution of 5\% chloral hydrate $(300 \mathrm{mg} / \mathrm{kg})$ was used to anesthetize the rats followed by tracheotomy. The arteria cervicalis and jugular vein of the rats were punctured and a catheter was embedded. Respiratory frequency, heart rate, systolic pressure, diastolic pressure, mean arterial blood pressure and central venous pressure were monitored. The rats were allocated at random into three groups $(\mathrm{n}=15) 30 \mathrm{~min}$ after attaining a stable state (Table I).

Specimen collection and preservation. Following establishment of the models, all the rats were sacrificed $24 \mathrm{~h}$ after initiation of the experiment. For each rat, the venous blood sample was collected to detect ADMA levels. Then, the chest was opened to expose the lungs. Following ligation of the right hilum, the upper-right lung was harvested and fixed with $4 \%$ paraformaldehyde. Hematoxylin and eosin (H\&E) staining of plastic-embedded tissue sections was examined by light microscopy (Motic BA 400; Motic, Xiamen, China).

Detection of plasma ADMA levels by enzyme linked immunosorbent assay (ELISA). The double sandwich ELISA method was used to measure plasma ADMA levels and was conducted according to the manufacturer's instructions (Shanghai Senxiong Biotech Industry Co., Ltd., Shanghai, China).

Detection of p38 MAPK/HSP27 by western blot analysis. Protein was extracted from the right lung lobe and preserved at $-80^{\circ} \mathrm{C}$ following detection of the protein concentration. The protein sample $(100 \mu \mathrm{g})$ was electrophoresed in $12 \%$ sodium dodecyl sulfate polyacrylamide gel electrophoresis (JY-JX5L; Beijing Jun Yi Huaxin Technology Co., Ltd., Beijing, China) and then transferred onto polyvinylidene fluoride membranes (Kynar K760; Inc., Philadelphia, PA, USA) using a constant voltage $(120 \mathrm{~V})$ at $4^{\circ} \mathrm{C}$. The membranes were sealed with $5 \%$ skimmed milk powder at room temperature $\left(22^{\circ} \mathrm{C}\right)$ for $1 \mathrm{~h}$.

The polyclonal p38MAPK (threonine 180 and tyrosine 182; 1:1,000; cat. no. AM065) and polyclonal p-HSP27 primary antibodies (1:1,000 dilution; cat. no. AH728) and GAPDH (an internal standard) primary antibody (1:500 dilution; cat. no. AG019). All antibodies were purchased from Beyotime Institute of Biotechnology (Shanghai, China) were mixed with the solution. Following overnight incubation at $4^{\circ} \mathrm{C}$, horseradish peroxidase-labeled rabbit anti-mouse secondary antibody (Sigma-Aldrich, St. Louis, MO, USA) at a dilution of 1:1,000 was added into the mixture, which caused a color change following incubation at room temperature for $1 \mathrm{~h}$. Images were captured using photographic photometry on an Exilim EX-FC100 digital camera (Casio, Shibuya, Japan) and the experimental results were analyzed using Quantity One Software (Bio-Rad Laboratories, Hercules, CA, USA). The optical density value was scanned and presented as the ratio of protein-to-internal standard GAPDH.
Immunofluorescent detection of globular actin (G-actin) and filamentous actin (F-actin). Following incubation for $2 \mathrm{~h}$, dewaxing in xylene (Sigma-Aldrich) and rehydrating in ethanol (Sigma-Aldrich), the sections were rinsed three times (5 min each time) with $30 \mathrm{ml}$ phosphate-buffered saline (PBS; pH 7.4; Gibco-BRL, Carslbad, CA, USA) with $1 \mathrm{mg} / \mathrm{ml}$ glucose at $25^{\circ} \mathrm{C}$. Microwave antigen retrieval was conducted subsequently using a microwave oven, microwave with pressure cooker and $0.01 \mathrm{M}$ sodium citrate buffer ( $\mathrm{pH}$ 6.0), followed by incubation for $1 \mathrm{~h}$ with blocking solution (buffer 1 containing $0.3 \%$ Triton X-100/2\% goat serum; Sigma-Aldrich) after rinsing three times with PBS. G-actin was stained with deoxyribonuclease I (DNase I; $0.3 \mu \mathrm{M}$ DNase I; Sigma-Aldrich). For F-actin staining, coverslips were incubated in a $10 \mathrm{U} / \mathrm{ml}$ solution $(0.165 \mathrm{M})$ of fluorescein isothiocyanate-labeled phalloidin (Sigma, St. Louis, MO, USA). After rinsing with PBS, tissues were bathed in $200 \mu 1$ 4', 6-diamidino-2-phenylindole (DAPI; Roche Diagnostics, Mannheim, Germany) for at least $5 \mathrm{~min}$. Green and red fluorescence protein indicated G-actin and F-actin, respectively. The nucleus showed blue fluorescence. The samples were investigated and images were captured using a fluorescence microscope (Motic BA 400T). The images were processed using digital analysis software Motic Fluo 1.0.

Statistical analysis. Continuous variables were expressed as the mean \pm standard deviation. Categorical data were presented as the frequencies and percentages. Differences among the groups were compared using the two-tailed, non-paired Student's t-test or one-way analysis of variance for continuous variables, where appropriate. Comparisons of categorical variables among groups were performed using the $\chi^{2}$ test. All tests of statistical significance were two-sided, with $\mathrm{P}<0.05$ being considered to indicate a statistically significant difference. Statistical analyses were performed using SPSS version 17.0 (SPSS, Inc., Chicago, IL, USA).

\section{Results}

Plasma ADMA levels and pulmonary permeability index(PI). Compared with the control group $(0.69 \pm 0.13 \mu \mathrm{mol} / \mathrm{l})$, the AKI $(1.93 \pm 0.17 \mu \mathrm{mol} / \mathrm{l})$ and AKI + SB203580 $(1.33 \pm 0.09 \mu \mathrm{mol} / \mathrm{l})$ groups demonstrated significant increases in plasma ADMA levels (all $\mathrm{P}<0.05$ ). There was also a significant difference in plasma ADMA levels between the AKI and AKI + SB203580 groups $(\mathrm{P}<0.05$; Fig. 1$)$. The increase in the $\mathrm{PI}$ in the AKI group $(6.54 \pm 0.42)$ was significantly higher than that in the control group $(1.91 \pm 0.16 ; \mathrm{P}<0.001)$. Significant differences in permeability were also found between the AKI group (6.54 \pm 0.42$)$ and the AKI + SB203580 group (3.24 \pm 0.1 ; $\mathrm{P}=0.027)$. However, no significant difference in PI was identified between the control group and the AKI + SB203580 group ( $\mathrm{P}=0.130$; Fig. 2).

Activation of 38 MAPK and HSP27. The expression of p-p38 and p-HSP27 was significantly higher in the AKI and AKI + SB203580 groups compared with the control group (all $\mathrm{P}<0.05)$. The AKI group also demonstrated a higher expression of p-p38 and p-HSP27 than the AKI + SB203580 group (all $\mathrm{P}<0.05$; Figs. 3 and 4 ). 
Table I. Experimental interventions performed on the rats in each group $(n=15)$.

Group Experimental method

Control

A midline abdominal incision was performed, bilateral renal artery and renal veins were separated without ligation; all rats received $2 \mathrm{ml}$ saline by intraperitoneal injection.

AKI

A midline abdominal incision was performed, bilateral renal artery and renal veins were separated with continuous occlusion; all rats received $2 \mathrm{ml}$ saline by intraperitoneal injection.

AKI + SB203580 A midline abdominal incision was performed, bilateral renal artery and renal veins were separated with continuous occlusion; all rats received $2 \mathrm{ml}(2 \mathrm{mg} / \mathrm{kg}$ ) SB203580 (p38 mitogen-activated protein kinase inhibitor) by intraperitoneal injection.

AKI, acute kidney injury.

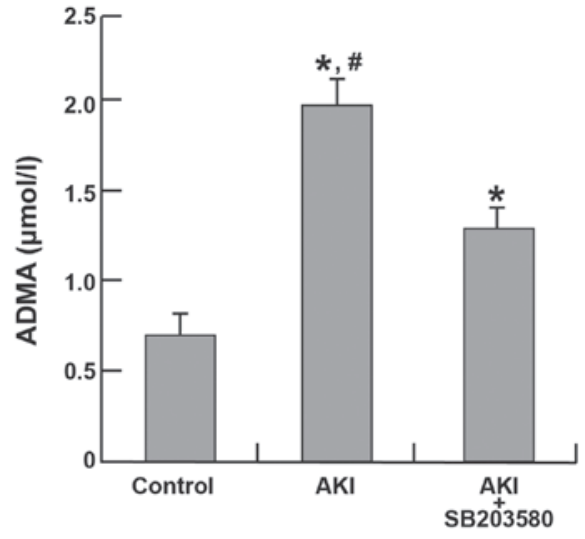

Figure 1. Plasma ADMA levels in the three groups. " $\mathrm{P}<0.05$ compared with the control group; ${ }^{*} \mathrm{P}<0.05$ compared with the AKI + SB203580 group. ADMA, asymmetric dimethylarginine; AKI, acute kidney injury.

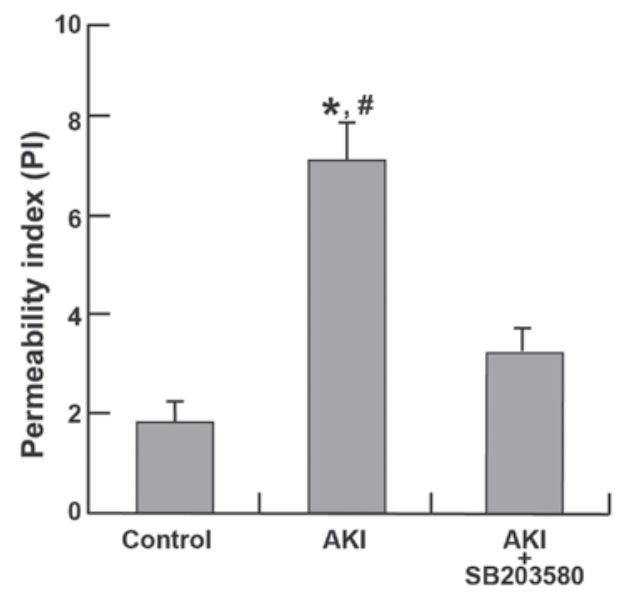

Figure 2. Permeability index in the three groups. ${ }^{*} \mathrm{P}<0.05$ compared with the control group; ${ }^{*} \mathrm{P}<0.05$ compared with the $\mathrm{AKI}+\mathrm{SB} 203580$ group. AKI, acute kidney injury.

Pathological changes of rat lung tissue. In the control group, rats exhibited good alveolar structural integrity without clear intra-alveolar exudation and interstitial pulmonary edema (Fig. 5A). By contrast, rats in the AKI group showed epithelial swelling, marked widening of alveolar walls due to capillary proliferation and congestion, which led to clear interstitial

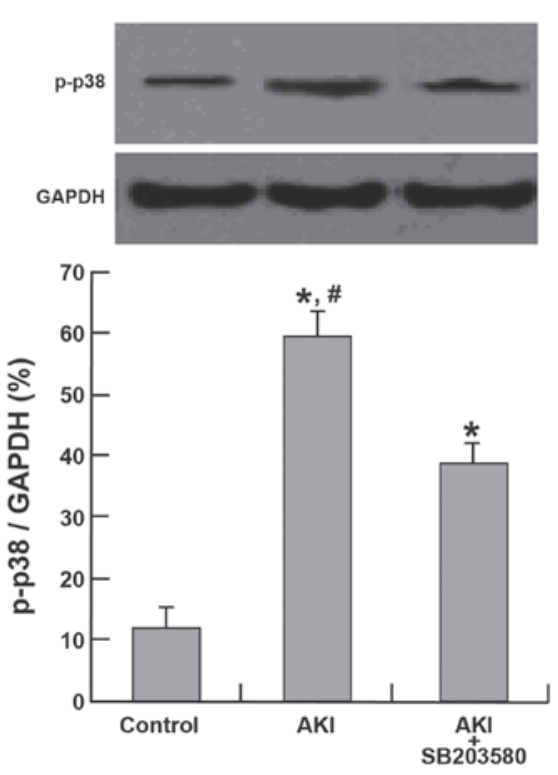

Figure 3. Expression of p-p38 in the three groups. "P $<0.05$ compared with the control group; ${ }^{~} \mathrm{P}<0.05$ compared with the AKI + SB203580 group. AKI, acute kidney injury; p-p38, phosphorylated p38.

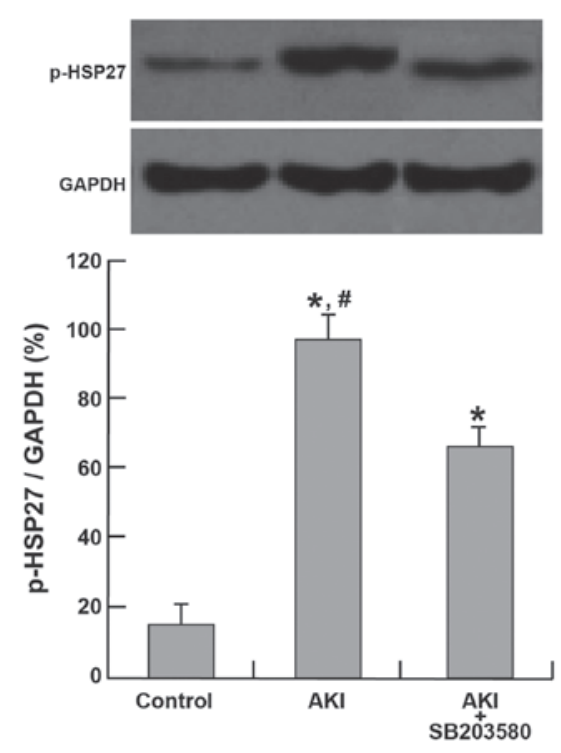

Figure 4. Expression of p-HSP27 in the three groups. " $\mathrm{P}<0.05$ compared with the control group; ${ }^{\text {P }}<0.05$ compared with the AKI $+\mathrm{SB} 203580$ group. AKI, acute kidney injury; p-HSP27, phosphorylated heat shock protein 27. 

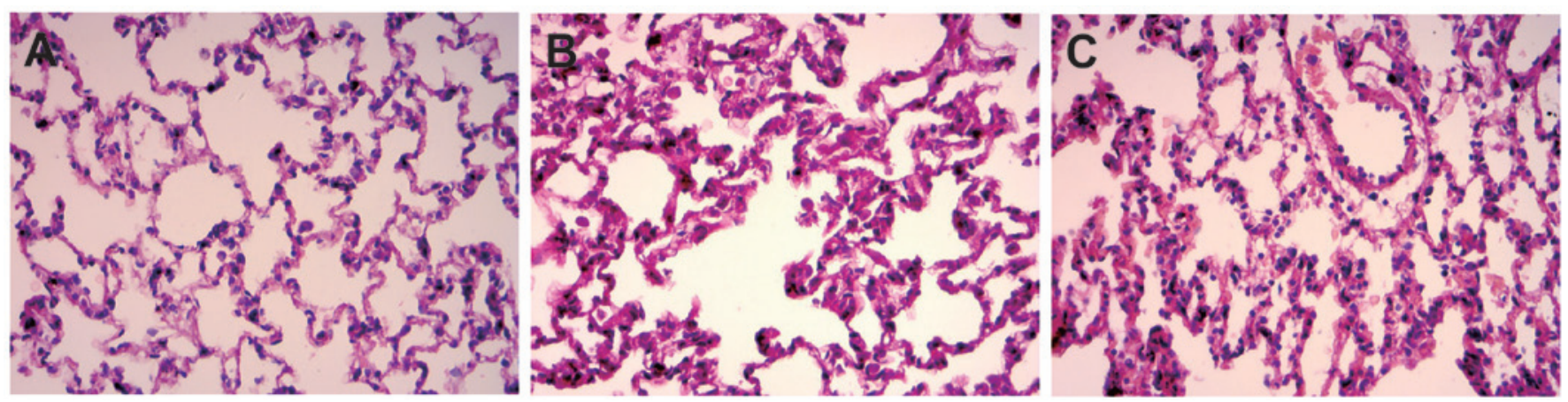

Figure 5. Pathological changes of rat lung tissue in the three groups (hematoxylin and eosin staining, magnification, $\mathrm{x} 400$ ). (A) the control group; (B) the AKI group and (C) the AKI + SB203580 group. AKI, acute kidney injury; F-actin, filamentous actin; G-actin, globular actin.
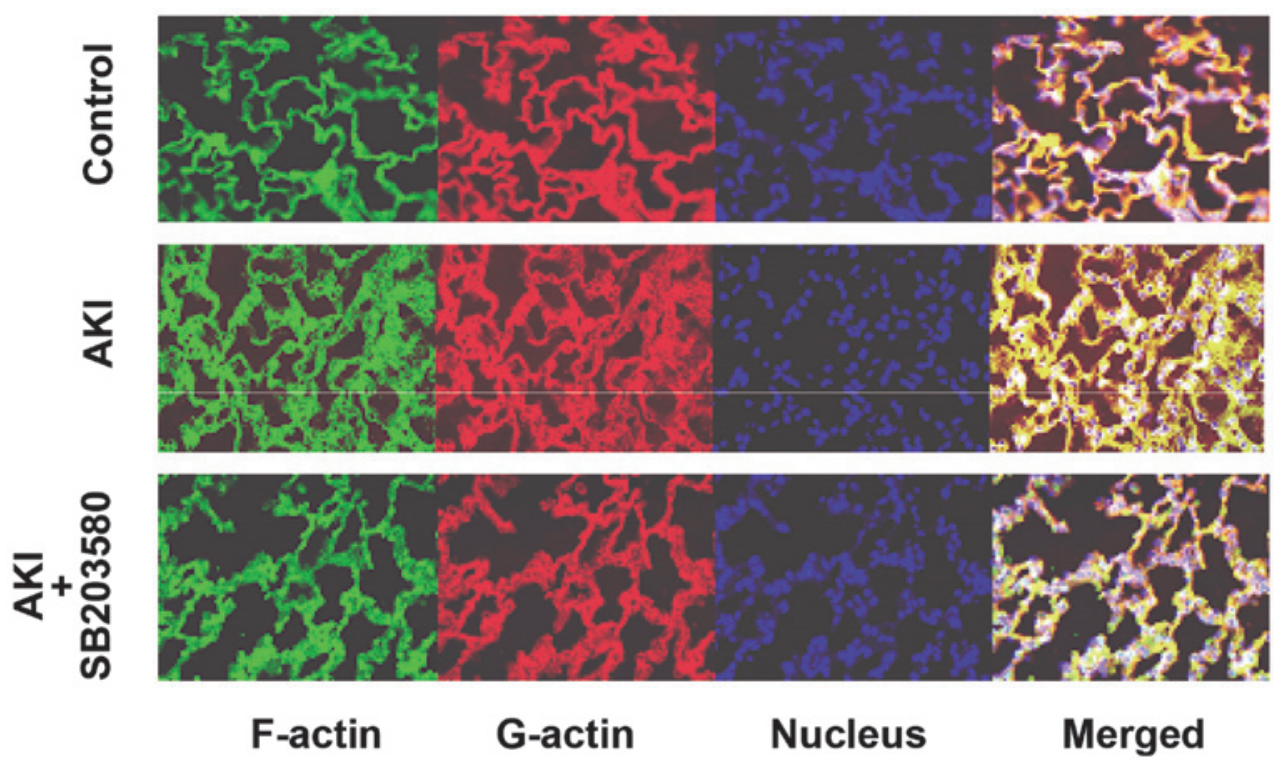

Figure 6. Immunofluorescence analyses of F-actin and G-actin in the three groups. The expression of F-actin in the AKI group was clearer than that in the control and AKI + SB203580 groups.

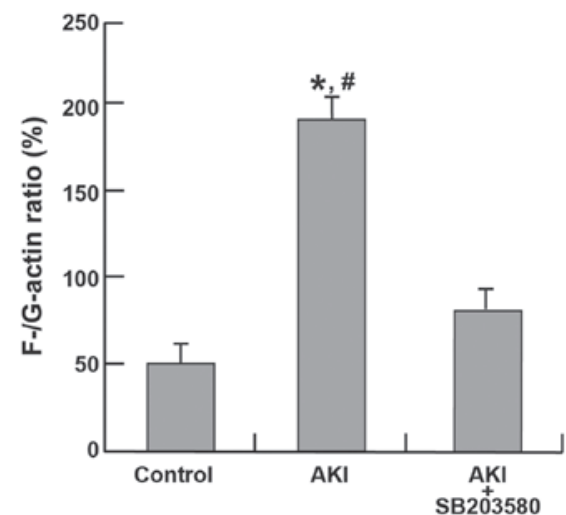

Figure 7. The F-/G-actin ratio in the AKI group was also higher compared with that of the control and AKI + SB203580 groups. AKI, acute kidney injury; F-actin, filamentous actin; G-actin, globular actin.

pulmonary edema with exudations of inflammatory cells, red blood cells and associated proteins, and conversely causing inflammatory cell infiltration (Fig. 5B). Small airway injury and alveolar structural disorder were also dientified in the AKI group. All the described symptoms demonstrate the pathological changes of acute lung injury (ALI). In the AKI + SB203580 group, rats also had exudations of inflammatory cells, red blood cells and associated proteins. However, compared with the AKI group, these rats demonstrated mitigations of edema (Fig. 5C).

Alterations in the F-/G-actin ratio. Immunofluorescence analyses of F-actin and G-actin are shown in Fig. 6. The expression of F-actin in the AKI group was clearer than that in the control and AKI + SB203580 groups. The F-/G-actin ratio in the AKI group was also higher compared with that of the control and AKI + SB203580 groups (all P<0.05; Fig. 7).

\section{Discussion}

AKI is a multifactorial syndrome and may be accompanied by multiple organ injury, including ALI or acute respiratory distress syndrome, particularly in critically ill patients $(15,16)$. Patients with AKI who are treated with RRT have a high mortality rate of $50-60 \%$ (17). It is well established that AKI is associated with a high mortality rate and distant organ dysfunctions. The current theory is that AKI 
induces pathological alterations of the lung, which predispose patients to a poor prognosis (2). The lung is the most easily damaged by ischemic injury due to the extensive vascular network in the lung mesenchyme (7). Findings of previous studies have indicated that ICU patients with AKI-induced ALI have a mortality rate of $\sim 80 \%$ (18). In a previous study, it was found that ALI usually occurs during the early stages of AKI, characterized by inflammatory cell infiltration, hyperemia in alveolar space and alterations in the structure of pulmonary alveoli (19). Microscopically, evidence of pulmonary endothelial injury was observed, including endothelial cell swelling, widening of interendothelial junctions and an increased number of pinocytotic vesicles.

Impaired epithelial barrier integrity and function are important features of ALI (20). It has been demonstrated that endothelial cell structure is maintained by the cytoskeleton, which is possibly responsible for maintaining and restoring normal cell morphology and functions (21). The actin microfilaments, which are mainly composed of F-actin and actin-binding proteins, are a major component of the cytoskeleton and are intricately involved mechanically and biochemically in various cell processes, including cell division and motility (22). It is well established that the polymerization-depolymerization dynamics of actin is a crucial process in a wide variety of cellular functions (23). HSP27, the F-actin polymerization modulator, is important in the reorganization of the actin cytoskeleton network of cells (24). It has been demonstrated that non-phosphorylated HSP27 was able to promote the reduction of F-actin in cells, thereby inhibiting F-actin polymerization (25). Activation of $\mathrm{p} 38$ caused the activation of MAPK-activated protein kinase-2/3 and phosphorylation of HSP27, leading to the F-actin polymerization and extension, and thereby inducing the recombination of the actin cytoskeleton and increase in intracellular space (26). These alterations may ultimately lead to the impairment of the barrier function of pulmonary endothelium and cause AKI.

In the present study, it was found that increased p-HSP27 may be associated with increases in the $\mathrm{F} / \mathrm{G}$-actin ratio and the degree of lung injury. It has been demonstrated that p-HSP27 was not able to inhibit F-actin polymerization and extension, which may promote the impairment of the integrity and dysfunction of the pulmonary endothelial and epithelial barrier (27). The present study also found that the expression levels of p-HSP27 demonstrated an expected positive correlation with p-p38 MAPK. The SB203580, an inhibitor of the p38 MAPK pathway, may decrease the expression levels of p-HSP27, indicating that $\mathrm{p} 38$ MAPK regulates the expression of p-HSP27.

The present study has demonstrated that ischemia-induced AKI rats exhibited significantly higher plasma ADMA levels than those in the control group. H\&E staining showed the typical pathological alterations of ALI, including alveolar inflammation, impairment of alveolar integrity and cell infiltration. Furthermore, p-p38MAPK, p-HSP27 and F-actin also progressively increased. Nevertheless, SB203580 was able to inhibit the expression of p-HSP27 and F-actin followed by reciprocal alleviation of ALI. A possible explanation may be that the activation of p38 MAPK downregulated the activity of HSP27, thereby inducing pulmonary vascular endothelial cell regeneration and reorganization. These alterations may affect cell permeability. The results suggested that the p38 MAPK/HSP27 signaling pathway is important in the development of AKI-induced ALI.

In conclusion, the results from the present study indicate that the plasma levels of ADMA may be a good biomarker for AKI-induced ALI. Furthermore, the p38 MAPK/HSP27 pathway is important in modulating the levels of ADMA.

\section{Acknowledgements}

The present study was supported by the Fund for Scientific Research of The First Hospital Of China Medical University (fsfh1103) and the Science and Technology project of Shenyang (F11-264-1-52).

\section{References}

1. Liu KD, Himmelfarb J, Paganini E, et al: Timing of initiation of dialysis in critically ill patients with acute kidney injury. Clin J Am Soc Nephrol 1: 915-919, 2006.

2. Himmelfarb J and Ikizler TA: Acute kidney injury: changing lexicography, definitions, and epidemiology. Kidney Int 71: 971-976, 2007.

3. Akcan-Arikan A, Zappitelli M, Loftis LL, et al: Modified RIFLE criteria in critically ill children with acute kidney injury. Kidney Int 71: 1028-1035, 2007.

4. Mehta RL, Kellum JA, Shah SV, et al: Acute Kidney Injury Network: report of an initiative to improve outcomes in acute kidney injury. Crit Care 11: R31, 2007.

5. Schwenger V, Weigand MA, Hoffmann O, et al: Sustained low efficiency dialysis using a single-pass batch system in acute kidney injury - a randomized interventional trial: the REnal Replacement Therapy Study in Intensive Care Unit PatiEnts. Crit Care 16: R140, 2012.

6. Martin A and Acierno MJ: Continuous renal replacement therapy in the treatment of acute kidney injury and electrolyte disturbances associated with acute tumor lysis syndrome. J Vet Intern Med 24: 986-989, 2010.

7. Kuchnicka K and Maciejewski D: Ventilator-associated lung injury. Anaesthesiol Intensive Ther 45: 164-170, 2013.

8. Hassoun HT, Grigoryev DN, Lie ML, et al: Ischemic acute kidney injury induces a distant organ functional and genomic response distinguishable from bilateral nephrectomy. Am J Physiol Renal Physiol 293: F30-F40, 2007.

9. White LE, Chaudhary R, Moore LJ, et al: Surgical sepsis and organ crosstalk: the role of the kidney. J Surg Res 167: 306-315, 2011

10. Böger RH: Asymmetric dimethylarginine, an endogenous inhibitor of nitric oxide synthase, explains the 'L-arginine paradox' and acts as a novel cardiovascular risk factor. J Nutr 134: 2842S-2847S, 2004.

11. Vallance $P$ and Leiper J: Cardiovascular biology of the asymmetric dimethylarginine:dimethylarginine dimethylaminohydrolase pathway. Arterioscler Thromb Vasc Biol 24: 1023-1030, 2004.

12. Palm F, Onozato ML, Luo Z, et al: Dimethylarginine dimethylaminohydrolase (DDAH): expression, regulation, and function in the cardiovascular and renal systems. Am J Physiol Heart Circ Physiol 293: H3227-H3245, 2007.

13. Fliser D, Kronenberg F, Kielstein JT, et al: Asymmetric dimethylarginine and progression of chronic kidney disease: the mild to moderate kidney disease study. J Am Soc Nephrol 16: 2456-2461, 2005.

14. Wang LY, Zhang DL, Zheng JF, et al: Apelin-13 passes through the ADMA-damaged endothelial barrier and acts on vascular smooth muscle cells. Peptides 32: 2436-2443, 2011.

15. Chertow GM, Burdick E, Honour M, et al: Acute kidney injury, mortality, length of stay, and costs in hospitalized patients. J Am Soc Nephrol 16: 3365-3370, 2005.

16. Togel F, Weiss K, Yang Y, et al: Vasculotropic, paracrine actions of infused mesenchymal stem cells are important to the recovery from acute kidney injury. Am J Physiol Renal Physiol 292: F1626-F1635, 2007. 
17. Hoste EA and Schurgers M: Epidemiology of acute kidney injury: how big is the problem? Crit Care Med 36: S146-S151, 2008.

18. Coca SG, Yusuf B, Shlipak MG, et al: Long-term risk of mortality and other adverse outcomes after acute kidney injury: a systematic review and meta-analysis. Am J Kidney Dis 53: 961-973, 2009

19. Ma T and Liu Z: Functions of aquaporin 1 and alpha-epithelial $\mathrm{Na}^{+}$ channel in rat acute lung injury induced by acute ischemic kidney injury. Int Urol Nephrol 45: 1187-1196, 2013.

20. Vadasz I, Raviv S and Sznajder JI: Alveolar epithelium and $\mathrm{Na}, \mathrm{K}-\mathrm{ATP}$ ase in acute lung injury. Intensive Care Med 33: 1243-1251, 2007.

21. Banan A, Choudhary S, Zhang Y, et al: Oxidant-induced intestinal barrier disruption and its prevention by growth factors in a human colonic cell line: role of the microtubule cytoskeleton. Free Radic Biol Med 28: 727-738, 2000.

22. Rosenthal KS, Perez R and Hodnichak C: Inhibition of herpes simplex virus type 1 penetration by cytochalasins B and D. J Gen Virol 66: 1601-1605, 1985
23. Fujiwara I, Takahashi S, Tadakuma H, et al: Microscopic analysis of polymerization dynamics with individual actin filaments. Nat Cell Biol 4: 666-673, 2002.

24. Weber NC, Toma O, Wolter JI, et al: Mechanisms of xenon- and isoflurane-induced preconditioning - a potential link to the cytoskeleton via the MAPKAPK-2/HSP27 pathway. Br J Pharmacol 146: 445-455, 2005.

25. Loktionova SA and Kabakov AE: Protein phosphatase inhibitors and heat preconditioning prevent Hsp27 dephosphorylation, F-actin disruption and deterioration of morphology in ATP-depleted endothelial cells. FEBS Lett 433: 294-300, 1998.

26. Rousseau S, Houle F, Landry J, et al: p38 MAP kinase activation by vascular endothelial growth factor mediates actin reorganization and cell migration in human endothelial cells. Oncogene 15: 2169-2177, 1997.

27. Xiong Z, Wang Y, Gong W, et al: Expression of Hsp27 correlated with rat detrusor contraction after acute urinary retention. Mol Cell Biochem 381: 257-265, 2013. 